\title{
$\mathrm{M}|\mathrm{R}| \mathrm{S}$ Internet Journal Nitride Semiconductor Research
}

\section{Influence of Poisson's ratio uncertainty on calculations of the bowing parameter for strained InGaN layers}

\author{
S. Stepanov ${ }^{1}$, W.N.Wang ${ }^{1}$, B.S.Yavich ${ }^{12}$, V. Bougrov ${ }^{2}$, Y.T.Rebane ${ }^{2}$ and Y.G.Shreter ${ }^{2}$ \\ ${ }^{1}$ University of Bath, \\ ${ }^{2}$ Ioffe Physical-Technical Institute,
}

(Received Friday, January 26, 2001; accepted Thursday, March 8, 2001)

\begin{abstract}
The composition dependence of emission energy of pseudomorphically strained InGaN layers with In content up to 0.2 is obtained. It is found that the main reason of "scatter" in published values of the InGaN bowing parameter is the uncertainty of the Poisson's ratio determination. It is shown that after recalculation to the same Poisson's ratio, most published data yield essentially the same results as compared to experimental uncertainty.
\end{abstract}

\section{Introduction}

Thin InGaN layers form the active region of most nitride-based light-emitting devices. The thickness of InGaN layers in these devices ranges from 3-5 $\mathrm{nm}$ in conventional quantum-well structures to $10-50 \mathrm{~nm}$ in charge asymmetric resonance tunneling structures [1]. The dependence of the emission wavelength on the In content in InGaN layers is important for device design. This dependence has been measured by many groups [2] [3] [4] [5] [6] [7] [8], and significantly "different" results have been obtained. The bowing parameter has been measured to be from $1 \mathrm{eV}$ to $3.2 \mathrm{eV}$ (see Table 1). The possible reasons for "scatter" in bowing parameter values have been widely discussed. It has been pointed out that determination of the In content by x-ray diffraction (XRD), using the linear interpolation of the $c$ lattice parameter between $\mathrm{GaN}$ and InN (Vegard's law), may result in a systematic overestimation of the In content, and low values of bowing parameter. This determination implicitly assumes that InGaN layers are relaxed. It was observed, however, that due to difficulty of dislocation formation at typical InGaN growth temperatures, InGaN layers with thickness much larger than the critical thickness remained pseudomorphic to GaN [2] [5] [9] [8] [10], and no misfit dislocations were formed at InGaN/GaN interface. Thus, the In content needs to be corrected with respect to elastic properties of the layers, resulting in higher values of the bowing parameter. The "scatter" in bowing parameter exists, however, even in this case. Direct measurement of the In content by Rutherford back-scattering spectrometry (RBS) [5] and secondary ion mass spectroscopy (SIMS) with cali- brated standards [8] confirms some of XRD results but differs significantly from others. Besides, it has been proven that the bowing parameter also depends on the method of the optical transition measurement. Photoluminescence (PL) [2] [3] [11] gives typically lower values of energy than optical transmission (OT) [4] [5], photothermal deflection spectroscopy (PDS) [7], photorefraction (PR) [6] [11] [8] and spectroscopic ellipsometry (SE) [12]. This difference, however, in addition to uncertainty in energy measurement due to possible In content fluctuations, is not large enough to explain "scatter" in the published values of bowing parameter.

In this paper, we report the $\operatorname{In}_{\mathrm{x}} \mathrm{Ga}_{1-\mathrm{x}} \mathrm{N}$ emission wavelength dependence on In content up to $\mathrm{x}=0.2$. We discuss the main reason for the "scatter" in measured values of bowing parameter for InGaN alloys. We estimate also the uncertainty of bowing parameter measurement. The main result is that no significant difference exists in bowing parameter values measured by various groups.

\section{Experimental}

Sets of samples have been grown by low pressure MOCVD on (0001)-oriented 2" sapphire rotated substrates. We used an AIX 200/4HT-S reactor equipped with in-situ optical reflectance monitoring for optimization of the growth parameters and control of the epilayer thickness. The growth pressure was 200 mbar. TMGa/ TEGa, TMIn, $\mathrm{NH}_{3}$ were employed as precursors and $\mathrm{H}_{2} / \mathrm{N}_{2}$ as gas carriers. Structures consisted of a $\mathrm{GaN}$ nucleation layer grown at low temperature, a $1.2 \mu \mathrm{m}-$ 
thick GaN buffer layer and upper $\operatorname{In}_{\mathrm{x}} \mathrm{Ga}_{1-\mathrm{x}} \mathrm{N}$ layers with thickness $15-70 \mathrm{~nm}$. Ternary alloys with $\mathrm{x}$ in the range $0.05-0.20$ were grown under temperatures in the range $700-840^{\circ} \mathrm{C}$.

The In content in the layers was determined using a high-resolution Bede D1 XRD system with $\mathrm{Cu} K \alpha_{1}$ radiation. The (00.2) reflections from $\mathrm{GaN}$ and (00.6) reflections from sapphire were measured to calculate the $c$-lattice constant of $\mathrm{GaN}$. The (00.6) reflections from $\mathrm{GaN}$ and $\mathrm{InGaN}$ were measured to calculate the $c$-lattice constant of InGaN. Despite lower x-ray intensity for these reflections compared to more typically measured (00.2) reflections, the accuracy of lattice constant measurements in this case is significantly higher. The inplane $a$-lattice constant was extracted from measurement of the asymmetrical (10.5), (20.5) and (11.4) reflections. The resulting $a$-lattice constants for the layer were calculated after averaging the values calculated from each of these three reflections. Room-temperature (RT) PL spectra were measured to obtain the emission wavelength of the InGaN layers. A $0.1-1 \mathrm{~mW} \mathrm{He}-\mathrm{Cd}$ laser was used as the excitation light source for PL.

\section{In content calculation}

For a strained InGaN layer on GaN, biaxial compressive strain $\varepsilon_{\|}$and the corresponding perpendicular strain $\varepsilon_{\perp}$ are induced in the layer, and

$$
\varepsilon_{\perp}(x)=-2 \frac{v(x)}{1-v(x)} \cdot \varepsilon_{\|}(x)
$$

where $v$ is the Poisson's ratio. For hexagonal layers grown along (00.1) axis the Poisson's ratio is expressed through elastic constants $C_{13}$ and $C_{33}$ as

$$
\frac{v(x)}{1-v(x)}=\frac{C_{13}(x)}{C_{33}(x)}
$$

Measuring the Poisson's ratio is difficult for InGaN films. For the first-order estimation of the In content in a strained or partially relaxed InGaN layer, the fixed Poisson's ratio can be assumed. Taking into account the dependence of the Poisson's ratio on the In content leads to more sophisticated estimation of the In content [10]. However, this estimation yields essentially the same results considering experimental uncertainty.

For calculation of the In content, it was assumed that Vegard's law is valid for both lattice constants of relaxed InGaN. The fixed relationship $c_{0} / a_{0}=1.629$ was assumed for relaxed $\mathrm{InGaN}$ and $\mathrm{GaN}$, according to the measured values of $c_{\mathrm{GaN}}=5.187 \AA$ and $a_{\mathrm{GaN}}=3.185 \AA$ for GaN. For relaxed $\mathrm{InN}$, the value of $c_{\mathrm{InN}}=5.705 \AA$ was assumed. The In content in the InGaN layers can then be calculated as

$$
x=\frac{1-v}{1+v} \cdot \frac{c-c_{\mathrm{GaN}}}{c_{\mathrm{InN}}-c_{\mathrm{GaN}}}+\frac{2 v}{1+v} \cdot \frac{a-a_{\mathrm{GaN}}}{a_{\mathrm{InN}}-a_{\mathrm{GaN}}}
$$

For InGaN layers pseudomorphic to $\mathrm{GaN}$, the second component of the sum can be neglected.

\section{Bowing parameter}

The emission energy of an $\operatorname{In}_{x} \mathrm{Ga}_{1-\mathrm{x}} \mathrm{N}$ alloy system is usually described by

$$
E(x)=(1-x) E_{\mathrm{GaN}}+x E_{\mathrm{InN}}-b \cdot x(1-x)
$$

where $b$ is a bowing parameter. The accuracy of bowing parameter measurements is limited by two main factors. The first is InGaN composition fluctuations over the sample surface. The second is the energy-position fluctuations in room-temperature PL measurements. We assume that both these factors have approximately the same influence on experimental accuracy, so that the uncertainty in emission-energy measurements is $\Delta E \approx$ $k T$. It gives the bowing parameter measurement uncertainty $\Delta b \geq 4 \Delta E \approx 0.1 \mathrm{eV}$. The uncertainty depends on maximal In content measured. The lower the In content, the higher the uncertainty. For example, for InGaN layers with maximal In content of only $0.10-0.12$, the uncertainty should be about $0.25 \mathrm{eV}$. For layers with maximal In content of 0.20 , the uncertainty should be about $0.15 \mathrm{eV}$.

\section{Poisson's ratio}

As it was mentioned above, the deformation of the unit cell in InGaN affects the interpretation of the In content from XRD measurements. The calculated In content depends on the Poisson's ratio. Accuracy required for the Poisson's ratio determination depends on the bowing parameter uncertainty. The admissible uncertainty of the In content calculation $\Delta x$ can be estimated from (4) as follows:

$$
\frac{\Delta x}{x} \approx-\frac{\Delta b}{E_{\mathrm{GaN}}-E_{\mathrm{InN}}+b}
$$

Thus, the admissible Poisson's ratio uncertainty for measuring the bowing parameter of strained InGaN alloys with In content up to 0.2 can be estimated from (3) and (5) as 


$$
|\Delta v| \approx \frac{1-v^{2}}{2} \cdot \frac{\Delta b}{E_{\mathrm{GaN}}-E_{\mathrm{InN}}+b} \approx 0.01 \div 0.02
$$

The values of the Poisson's ratio used by various groups in their calculations are listed in Table 1. It is evident that existing uncertainty in these values is higher than the admissible Poisson's ratio uncertainty. In several works, InGaN layers were assumed relaxed, formally corresponding to $v=0$ in (3). It results in low values of the bowing parameter. In other works, the strain in the layers is considered. In these works, however, there exists a scatter in values of the Poisson's ratio. The uncertainty results from the differences between theoretical and various experimental data on the elastic constants of GaN. Theoretical calculations [13] [14] predict the value of $2 C_{13} / C_{33}$ to be about 0.51 for GaN, leading to a Poisson's ratio of about 0.20. Calculation with this value yields, however, a lower In content compared to direct measurement by SIMS [8] or RBS [5]. The measured Poisson's ratio in the case of RBS In-content determination should be 0.18 . Several experimental works were performed for measuring the Poisson's ratio. Detchprohm et al. [15] reported $2 C_{13} /$ $C_{33}=0.38(v=0.16)$ after fitting their data on $c$ - and $a$ lattice constants for thick relaxed GaN layers on sapphire. Deger et al. [16] reported $2 C_{13} / C_{33}=0.56(v=$ 0.22 ) of GaN from the surface acoustic-wave measurements on epitaxial AlGaN films. Yamaguchi et al. [17] reported $2 C_{13} / C_{33}=0.60(v=0.23)$ from the Brillouin scattering study of epitaxial GaN films. This value was widely used by many authors for determination of the bowing parameter. However, as it was mentioned by Yamaguchi et al. [18], the measured elastic constants of thin $\mathrm{GaN}$ epitaxial films differ significantly from actual values due to the presence of residual stains in epitaxial layers. In addition, epitaxial films have high dislocation densities, invariability affecting properties of GaN. The Brillouin scattering study for bulk GaN [18] [19] gave $2 C_{13} / C_{33}=0.42(v=0.17)$.

As it is seen, the most reliable data on the Poisson's ratio measurements [5] [15] [18] [19] give the same results as compared to the admissible Poisson's ratio uncertainty. In our calculations, we use $v=0.17$ as an average of these data.

\section{Results}

Figure 4 presents the measured emission energy dependence on the In content in the InGaN layers. Error bars represent the possible In content range (as measured from $c$-lattice constant only) depending on the relaxation degree of the InGaN layers. The right end of the bar corresponds to fully relaxed material and the left end corresponds to fully strained material. This curve also illustrates possible errors in In content determination when calculations do not assume the strain in the layers (model of relaxed material). The same data in comparison with previously published data are presented on Figure 5. On Figure 6, the published data are recalculated with respect to the same Poisson's ratio $v=0.17$. All curves are corrected also to the same GaN emission energy of $3.42 \mathrm{eV}$. The curves show that all the data on InGaN emission energy (band-gap) vs. In content agree well in the framework of the admissible bowing parameter uncertainty discussed above. Additional changes in measured dependence can be made after correcting to the same lattice parameters of $\mathrm{GaN}$ and $\mathrm{InN}$.

\section{Conclusion}

The RT emission energy dependence on In content ( $c$ lattice constant) of pseudomorphically strained $\mathrm{InGaN}$ layers grown on $\mathrm{GaN}$ is measured. It is shown that the existing "scatter" in various published data on this dependence originates mostly from strong uncertainty in the values of the (In)GaN Poisson's ratio used. After correcting to the same Poisson's ratio value, all published data on the bowing parameter in strained InGaN layers yield essentially the same results considering the experimental uncertainty in emission energy and lattice constant measurements.

\section{ACKNOWLEDGMENTS}

The work was supported by Arima Optoelectronic Co. (UK). This is gratefully acknowledged.

\section{REFERENCES}

[1] Y. T. Rebane, Y. G. Shreter, B. S. Yavich, V. E. Bougrov, S. I. Stepanov, W. N. Wang, Phys. Stat. Sol. A 180, 121 (2000).

[2] T Takeuchi, H Takeuchi, S Sota, H Sakai, H Amano, I Akasaki, Jpn. J. Appl. Phys. 36, L177 (1997).

[3] S. Nakamura, Sol. St. Comm. 102, 237-248 (1997).

[4] M. D. McCluskey, C. G. Van de Walle, C. P. Master, L. T. Romano, N. M. Johnson, Appl. Phys. Lett. 72, 2725 (1998).

[5] L. T. Romano, B. S. Krusor, M. D. McCluskey, D. P. Bour, K. Nauka, Appl. Phys. Lett. 73, 1757 (1998).

[6] C Wetzel, T Takeuchi, S Yamaguchi, H Katoh, H Amano, I Akasaki, Appl. Phys. Lett. 73, 1994-6 (1998).

[7] F. Scholz, J. Off, A. Sohmer, V. Syganow, A. Dörnen, O. Ambacher, J. Cryst. Growth 189/190, 8 (1998).

[8] J. Wagner, A. Ramakrishnan, D. Behr, M. Maier, N. Herres, M. Kunzer, H. Ogloh, K-H. Bachem, MRS Internet J. Nitride Semicond. Res. 4S1, G2.8 (1999).

[9] J. Off, A. Kniest, C. Vorbeck, F. Scholz, O. Ambacher, J. Cryst. Growth 195, 286 (1998).

[10] L. Gorgens, O. Ambacher, M. Stutzmann, C. Miskys, F. Scholz, J. Off, Appl. Phys. Lett. 76, 577 (2000). 
[11] Christian Wetzel, Shugo Nitta, Tetsuya Takeuchi, Shigeo Yamaguchi, H. Amano, I. Akasaki, MRS Internet J. Nitride Semicond. Res. 3, 31 (1998).

[12] J. Wagner, A. Ramakrishnan, D. Behr, H. Obloh, M. Kunzer, K. -H. Bachem, Appl. Phys. Lett. 73, 1715 (1998).

[13] Kwiseon Kim, Walter R. L. Lambrecht, Benjamin Segall , Phys. Rev. B 53, 16310-16326 (1996).

[14] AF Wright, J. Appl. Phys. 82, 2833-2839 (1997).

[15] T Detchprohm, K Hiramatsu, K Itoh, I Akasaki, Jpn. J. Appl. Phys. 31, L1454-L1456 (1992).

[16] C. Deger, E. Born, H. Angerer, O. Ambacher, M. Stutzmann, J. Hornsteiner, E. Riha, G. Fischerauer, Appl. Phys. Lett. 72, 2400 (1998).

[17] M. Yamaguchi, T. Yagi, T. Azuhata, T. Sota, K. Suzuki, S. Chichibu, S. Nakamura, J. Phys. C 9, 241 (1997).

[18] M. Yamaguchi, T. Yagi, T. Sota, T. Deguchi, K. Shimada, S. Nakamura, J. Appl. Phys. 85, 8502 (1999).

[19] T. Deguchi, D. Ichiryu, K. Toshikawa, K. Sekiguchi, T. Sota, R. Matsuo, T. Azuhata, T. Yagi, S. Chichibu, S. Nakamura, J. Appl. Phys. 86, 1860 (1999).

\section{FIGURES}

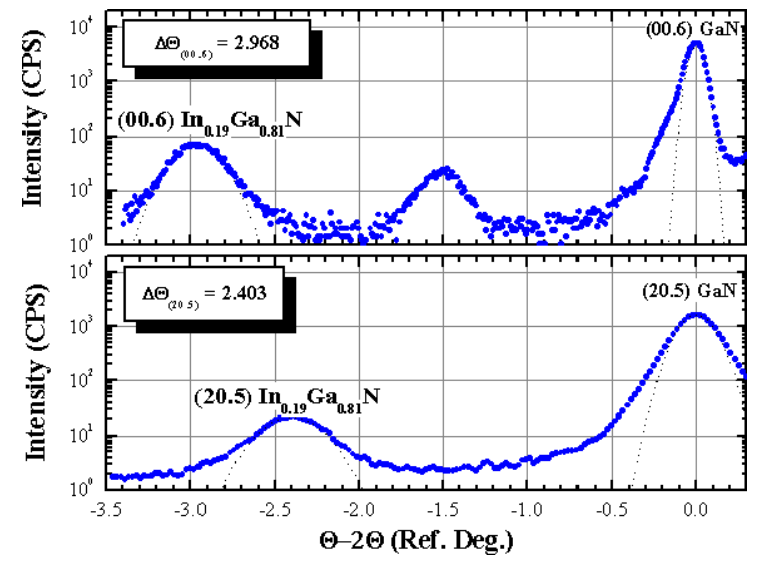

Figure 1. XRD rocking curves of symmetric (00.6) and asymmetric (20.5) reflections for $40 \mathrm{~nm}$ - thick $\mathrm{In}_{0.19} \mathrm{Ga}_{0.81} \mathrm{~N}$ layer grown on $\mathrm{GaN}$.

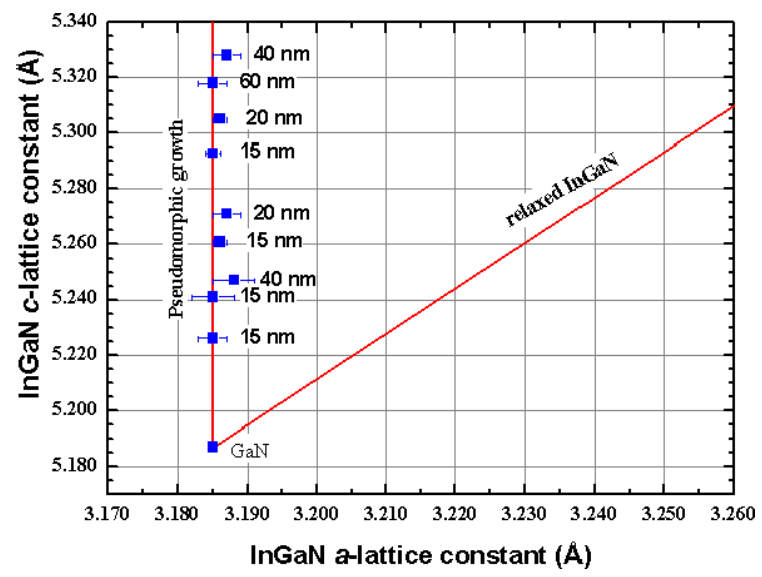

Figure 2. $c$-lattice constant vs. $a$-lattice constant for the InGaN layers on GaN. The vertical and dashed line marks GaN $a$ lattice parameter. The diagonal dashed line represent lattice constants dependence for relaxed InGaN with Vegard's law validity assumption.

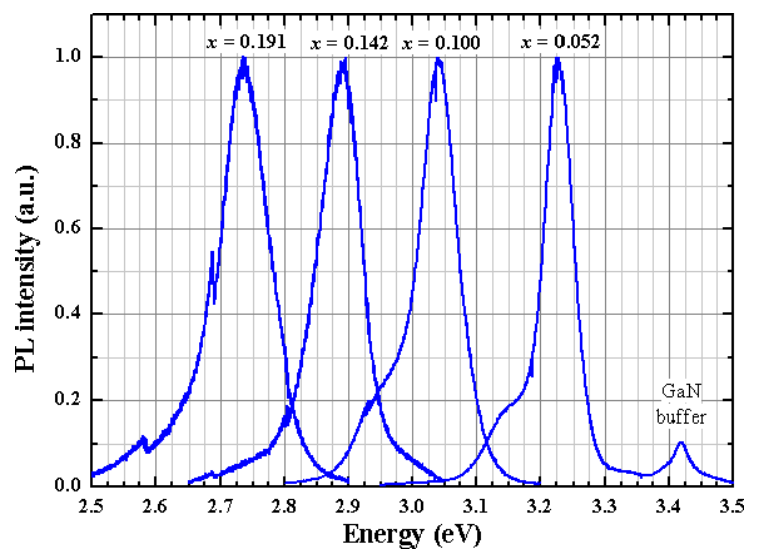

Figure 3. Room-temperature PL spectra for the InGaN layers grown on $\mathrm{GaN}$. 


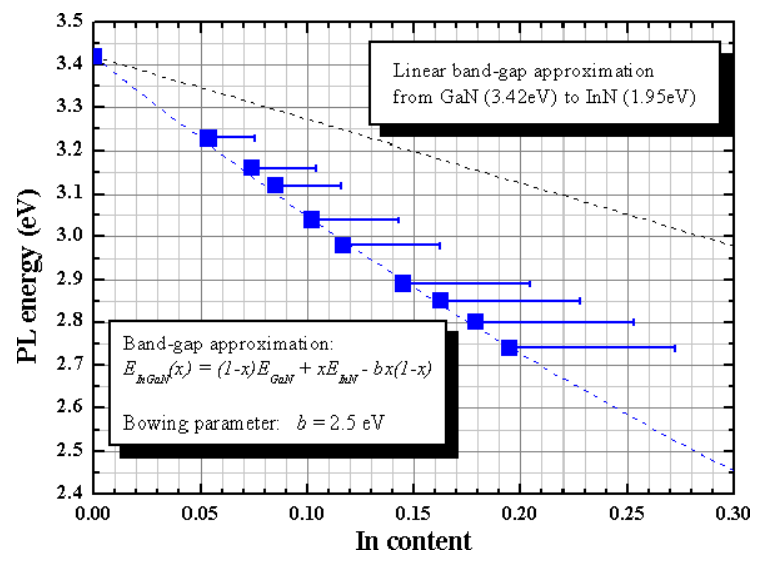

Figure 4. Measured emission energy dependence on the In content in the InGaN layers. Error bars represent the possible In content range (as measured from $c$-lattice constant) depending on the relaxation degree of the $\mathrm{InGaN}$ layers. Maximal In content in error bars corresponds to fully relaxed InGaN layers (with misfit dislocations), while minimal In content corresponds to InGaN layers pseudomorphic to $\mathrm{GaN}$.

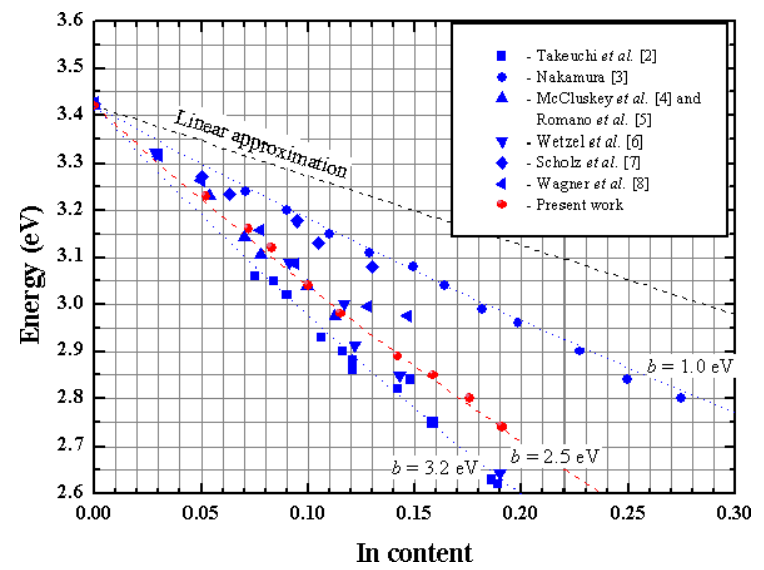

Figure 5. Emission energy dependence on the In content in the InGaN layers. "As is" published data are shown.

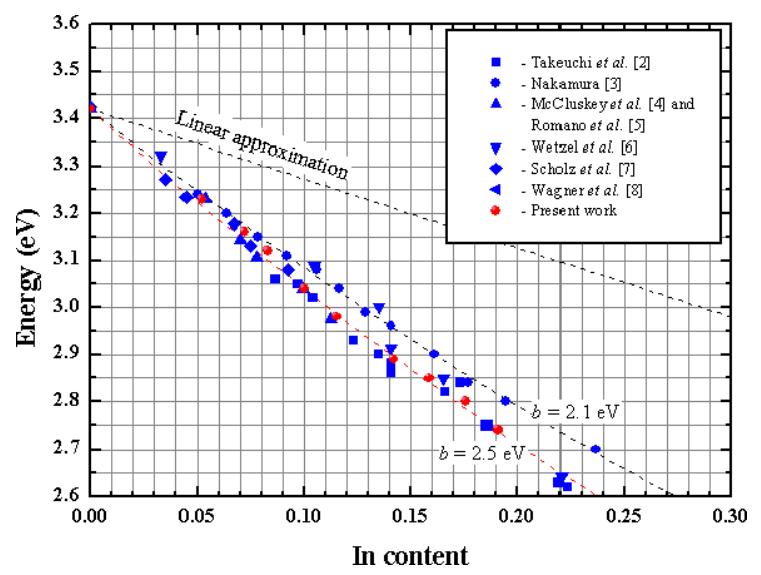

Figure 6. Emission energy dependence on the In content in the InGaN layers. Recalculated with the same Poisson's ratio published data are shown. 


\section{TABLES}

Table 1. Values of bowing parameter for strained InGaN layers obtained by various groups. Recalculated values with consideration of the same Poisson's ratio $v=0.17\left(2 C_{13} / C_{33}=0.41\right)$ are also given. For references [2] and [6] the Poisson's ratio is calculated from the given value of $2 C_{13} / C_{33}$. For references [7] and [8] the bowing parameter is calculated from given experimental points.

\begin{tabular}{|c|c|c|c|c|c|c|}
\hline & Composition & Poisson's ratio & In content & Energy & \multicolumn{2}{|c|}{ Bowing parameter $(\mathrm{eV})$} \\
\hline & & & & & measured & recalculated \\
\hline Present work & XRD & 0.17 & $0-0.2$ & $\mathrm{PL}$ & 2.5 & 2.5 \\
\hline Takeuchi et al. [2] & XRD & $0.23+0.10 \cdot x$ & $0-0.2$ & $\mathrm{PL}$ & 3.2 & 2.6 \\
\hline Nakamura [3] & XRD & 0 & $0-0.33$ & $\mathrm{PL}$ & 1.0 & 2.1 \\
\hline $\begin{array}{l}\text { McCluskey et al. [4] and } \\
\text { Romano et al. [5] }\end{array}$ & RBS \& XRD & $\begin{array}{l}(0.18) \\
\end{array}$ & $0-0.11$ & OT & 2.5 & - \\
\hline \begin{tabular}{|l} 
Wetzel et al. [6] \\
\end{tabular} & XRD & $0.23+0.05 \cdot x$ & $0-0.2$ & PR & 2.6 & 2.1 \\
\hline & & & & $\mathrm{PL}$ & 3.2 & 2.6 \\
\hline Scholz et al. [7] & XRD & 0 & $0-0.13$ & PDS & 1.1 & 2.2 \\
\hline 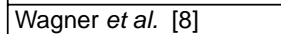 & SIMS & - & $0-0.15$ & $\mathrm{PR}$ & 2.0 & - \\
\hline
\end{tabular}

